Año LII urtea

N. ${ }^{\circ}$ 94. zk.

2020

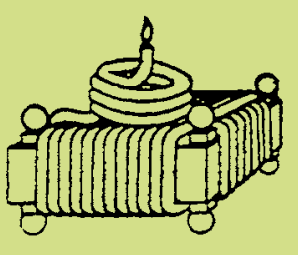

\section{CUADERNOS de Etnología y Etnografía de Navarra}

SEPARATA

\title{
Joxe Ulibarrena y su pasión por la Etnografía
}

Elur Ulibarrena Herce 


\section{Sumario / Aurkibidea}

\section{Cuadernos de Etnología y Etnografía de Navarra}

Año LII urtea - N. ${ }^{\circ}$ 94. zk. - 2020

\section{ARTÍCULOS/ARTIKULUAK}

Etxe izen zizelkatuak. Ageriko idazkunak etxeetan eta hilobietan

Koldo Colomo Castro

Recardera, oficio de mujer

Ricardo Gurbindo Gil

Aralar mugarriturik (1523-1857)

Jose Luis Erdozia Mauleon

El lecayo, una soka-dantza del siglo XVI

Ricardo Urrizola Hualde

El recuerdo público de una muerte política: historiografía periodística y etnografía de la ritualidad pública (José Luis Cano Pérez, 1949-1977)

Kepa Fernández de Larrinoa, Karlos Irujo Asurmendi, Santiago Martínez Magdalena, Txuri Ollo Gorriti, Cristina Saura Blanco

El Camino de Santiago en Navarra en 1971 a la luz de un informe para su mejora elaborado por la Dirección de Turismo, Bibliotecas y Cultura Popular Fernando Vega López

Reformulación del discurso significativo sociocultural en el entramado fortificado de la Línea P (Valle de Roncal-Navarra) 


\section{Sumario / Aurkibidea}

NOTICIAS/BERRIAK

Joxe Ulibarrena y su pasión por la Etnografía

Elur Ulibarrena Herce

Normas para la presentación de originales / Idazlanak aurkezteko arauak / 


\title{
Joxe Ulibarrena y su pasión por la Etnografía
}

\author{
Joxe Ulibarrena: etnografiarako grina
}

Joxe Ulibarrena and his passion for Ethnography

\author{
Elur ULIBARRENA HERCE \\ Museo Etnográfico del Reino de Pamplona \\ ulibarrenamuseo@gmail.com
}

DOI: https://doi.org/10.35462/CEEN94.8

Un personaje carismático, eso ha sido Joxe Ulibarrena en el mundo de la etnografía en Euskal Herria. Un artista de los que quedan en el recuerdo de la gente corriente.

Enamorado de su cultura y conocedor de su historia, autodidacta, emprendedor, hombre de palabra, entusiasta, constante y juguetón.

Inició su andadura en el mundo de la antropología después de estudiar Bellas Artes en París, durante su exilio en Venezuela en la década de 1950 de la mano del hermano Ginés y otros investigadores cercanos a los hermanos De la Salle.

Durante su inmersión en la vida cotidiana de diversas tribus del Amazonas, Joxe Ulibarrena tuvo la oportunidad de registrar como dibujante las prácticas culturales de aquellos grupos humanos que apenas habían tenido contacto con la cultura occidental, mientras aprendía de aquel estilo de vida.

Este aprendizaje empírico lo iba plasmando en bocetos y dibujos que le hicieron cambiar para siempre su estilo artístico. Los rasgos étnicos de la Virgen del Cambur o de la Virgen de Coromoto no son sino obras realizadas tras aquellas vivencias, completamente ligadas a la identidad y a la cultura oriunda.

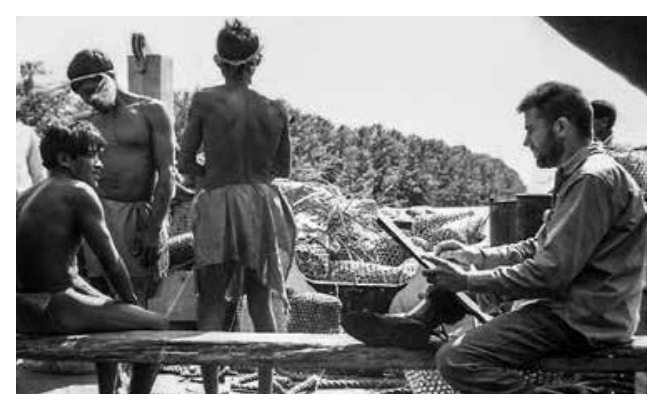

Figura 1. El primer contacto de Joxe Ulibarrena con el método etnográfico tuvo lugar con los pueblos nativos del delta del Orinoco, en Venezuela. 
Al regresar a Navarra, se estableció en la Casa Napartxo de Berrioplano, donde alternaba su trabajo como artesano, restaurador y anticuario con la producción artística.

Aquella inquietud por la cultura popular que había despertado en Sudamérica le llevó a recorrer los lugares más recónditos del territorio euskaldún y a aprender el idioma de sus antepasados. Zambullirse en Euskal Herria le ayudó a impregnarse de las historias de sus gentes, a conocer los modos de vida, las costumbres y todo aquello que hace de nuestra cultura eúskara una superviviente respecto a la cultura dominante.

Fue amigo personal de don José Miguel de Barandiarán, con quien compartía largas charlas antes y después de los cursos de cultura vasca que este impartía en la Universidad de Navarra; fue quien le impulsó a abrir al público una incipiente colección etnográfica, presentada a modo de museo en Casa Napartxo, en Berrioplano.

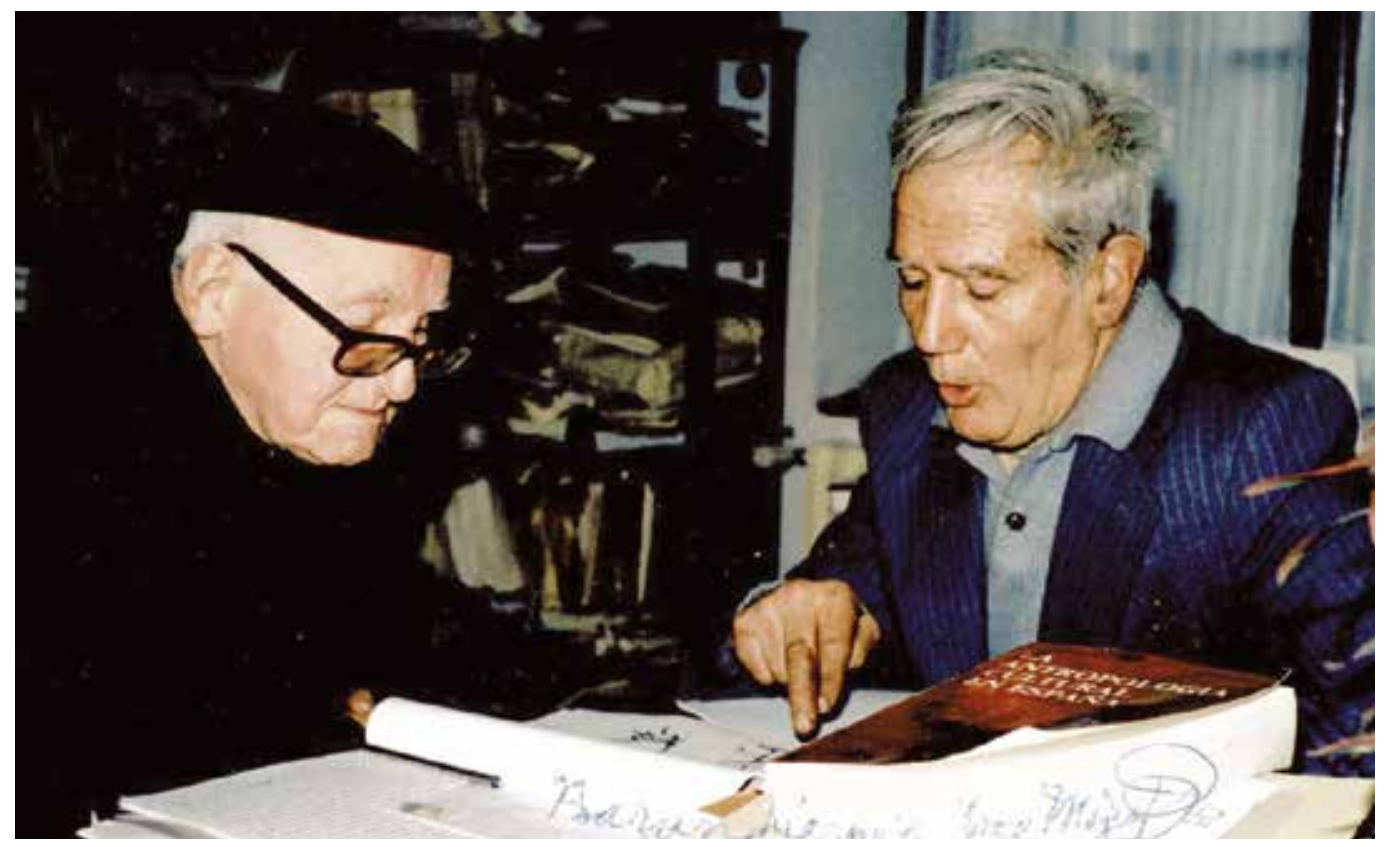

Figura 2. Ulibarrena en compañía de su maestro y amigo don José Miguel de Barandiaran.

Al poco tiempo creó la Fundación Mariscal Pedro de Navarra con el apoyo de toda la familia, con el objetivo de proteger y gestionar las colecciones del Museo Etnográfico que inauguró en 1982.

Sus ansias de que Navarra tuviera un museo etnográfico de titularidad pública no hicieron eco ni en Diputación ni tampoco en ningún Ayuntamiento. Ulibarrena aprendió que las élites raras veces se interesan por la cultura popular y frecuentemente se encargan de enturbiarla con exigencias que solo responden a los intereses de los partidos políticos. Así que decidió utilizar sus propios medios. 
Durante diez años se dedicó a acondicionar la casa Fantikorena de Arteta, donde tenía su taller, y en 1986 trasladó el museo al valle de Ollo.

A base de esfuerzo personal, familiar y mucho dinero consiguió hacer una colección etnográfica que reúne cerca de ocho mil piezas etnográficas procedentes del antiguo reino de Navarra.

Ulibarrena logró que su colección privada participara de la Red de Museos de Navarra y que la Fundación Mariscal Pedro de Navarra tuviera durante más de veinte años un convenio anual con la Institución Príncipe de Viana.

La gran mayoría de piezas que hoy forman parte del museo fueron adquiridas en caseríos recién abandonados en busca de una vida más próspera, a familias en proceso de deconstrucción, en momentos donde se pagaban a precio de chatarra.

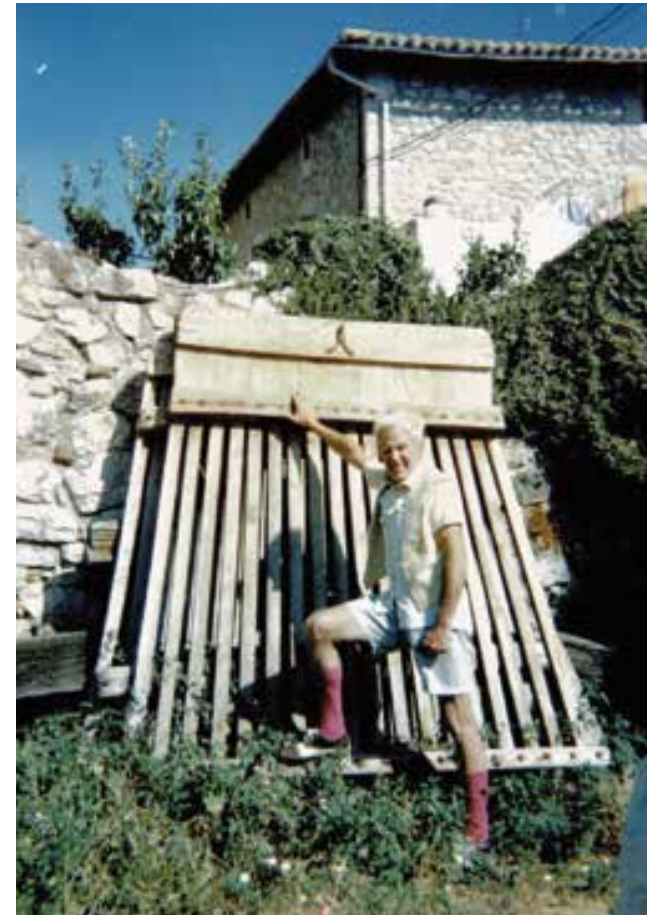

Figura 3. Joxe Ulibarrena en Arteta, lugar a donde trasladó el Museo Etnográfico del Reino de Pamplona en 1986.

Ulibarrena reflexionaba profundamente en torno a los objetos que forman parte de su amplia colección. Cada objeto fue el producto de una necesidad y de la reflexión en torno a esta; cada pieza fue la materialización de una idea ingeniosa, la solución innovadora para un problema que planteaba la vida. En cada obra artesanal hay un extenso conocimiento del oficio, experiencia y necesidad de innovar para la mejora de la comunidad. Esto pone de manifiesto que nuestras/os antepasadas/os, pese a no ser bien consideradas/os ni siquiera reconocidas/os socialmente, sino tachadas/os peyorativamente de aldeanas/os, eran muy inteligentes porque vivían en armonía con su ecosistema, cosa que no podemos decir la gran mayoría de nosotras/os, las/os «modernícolas» del siglo XXI, como nos llamaba Ulibarrena.

Cada pieza del museo fue elegida entre muchas otras, que en su día pasaron por las manos de Joxe. Las que no están hoy en la colección es que tuvo que revenderlas para comprar otras que a su criterio eran más adecuadas.

Pero Ulibarrena no dejó pasar la oportunidad de reconocer a las personas que las idearon, que las fabricaron y las usaron, que las conservaron y las vendieron. Extrajo de las historias que acompañan a cada herramienta la inspiración para sus esculturas, creando un estilo muy personal que brota de un conocimiento profundo de la trayectoria cultural de Euskal Herria y de la historia de Navarra. Sus pensamientos e ideas se plasmaron en varios libros, entre los que destacaré La étnica, plástica euskariana, o La España Neolítica, editados por la fundación. 


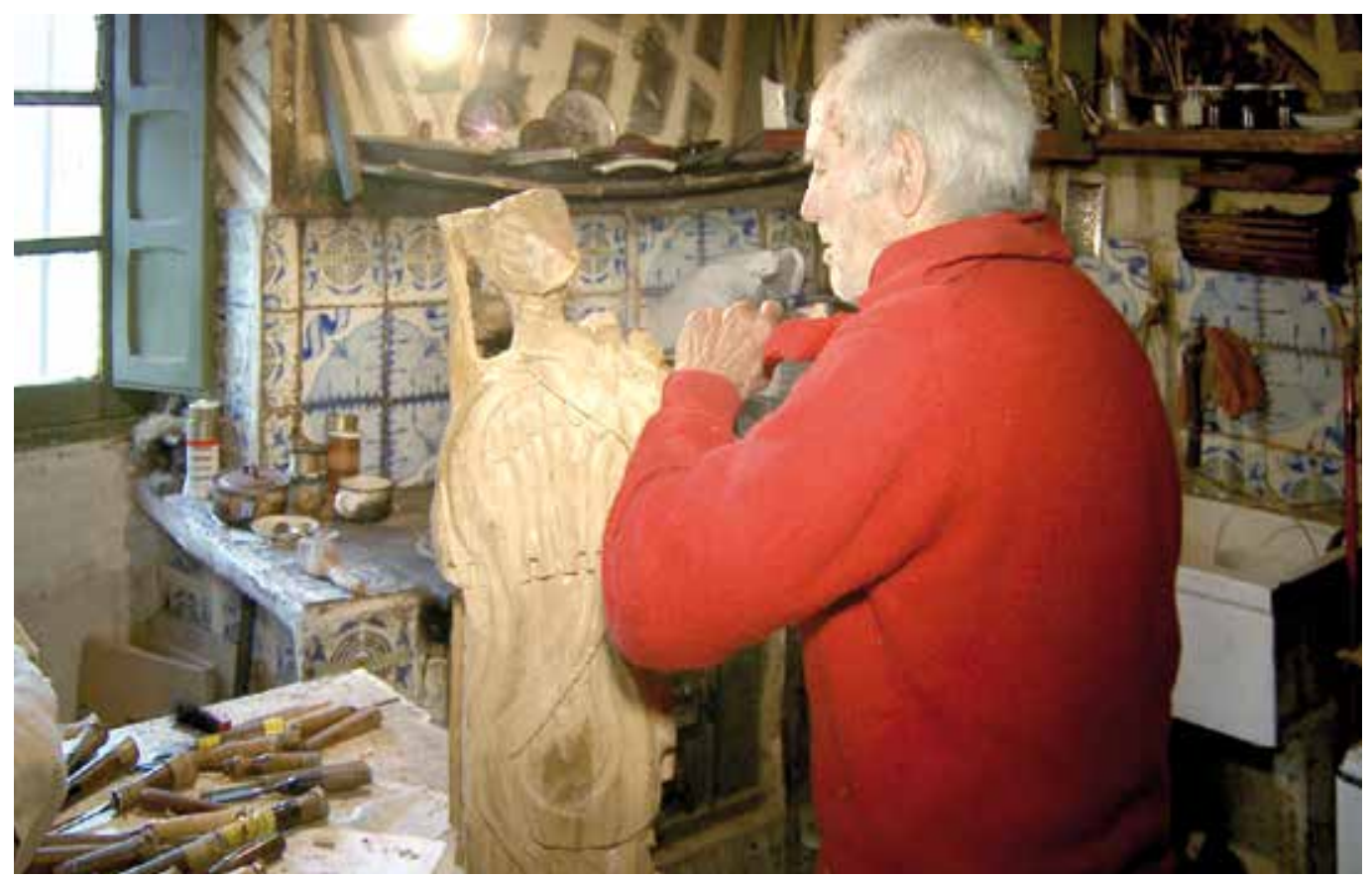

Figura 4. Trabajando en la escultura «Urepeleko artzaina» en su taller de Casa Fantikorena en 2014.

Los perfiles afilados que esculpe son los de aquellos antepasados que habitaron las aldeas, aldeanos y aldeanas que demostraron ser artistas en su gremio y que dando lo mejor de sus mentes y de sus manos aportaron progreso a sus comunidades, pero siguen siendo ninguneados.

La mirada que nos ofrece Ulibarrena es la del reconocimiento: no se trata de nostalgia del pasado, sino de dignificar lo pisoteado.

Su museo y su casa, Fantikorena, fue su último hogar, aquel que le arrebataron en su infancia, junto a su padre, en 1936. Hoy es el espacio donde se fusiona el legado de su pueblo con el suyo propio.

Entrar allí es como mirar al cielo e intentar ver todas las estrellas a la vez.

Goian bego aita!

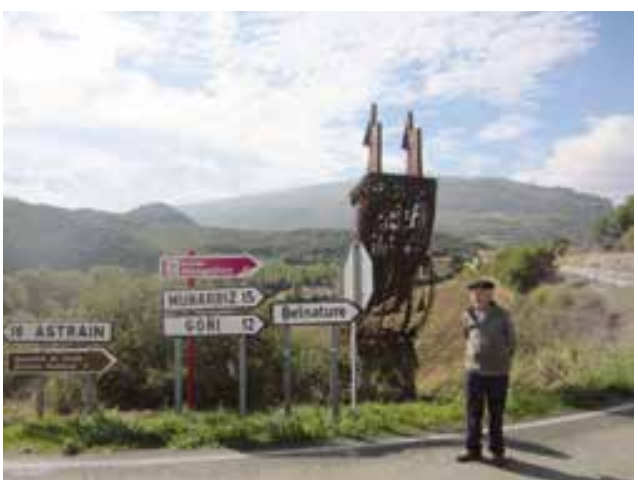

Figura 5. Joxe Ulibarrena junto a la escultura titulada "Centauros del Pirineo», creada en homenaje a Félix Urabayen y situada en la entrada del Valle de Ollo. 